\title{
Comparison of Kyoto Scale of Psychological Development and Bayley Scales of Infant Development second edition among Japanese Infants
}

\author{
Nozomi Tatsuta ${ }^{1,2, *}$, Keita Suzuki ${ }^{3}$, Tomoko Sugawara ${ }^{4}$, \\ Kunihiko NAKAI ${ }^{1}$, Toru Hosokawa ${ }^{2}$ and Hiroshi SATOH ${ }^{5}$ \\ ${ }^{1}$ Development and Environmental Medicine, Tohoku University Graduate School of Medicine, Japan \\ ${ }^{2}$ Human Development and Disabilities, Tohoku University Graduate School of Education, Japan \\ ${ }^{3}$ Faculty of Education, Kochi University, Japan \\ ${ }^{4}$ Department of Neuropsychiatry, Hirosaki University School of Medicine, Japan \\ ${ }^{5}$ Environmental Health Sciences, Tohoku University Graduate School of Medicine, Japan
}

\begin{abstract}
The Kyoto Scale of Psychological Development (KSPD), a standardized developmental assessment tool for Japanese infants, and the Bayley Scales of Infant Development second edition (BSID-II) have been widely used in clinical assessments and research on infants. The purpose of this study was to compare the psychometric indices of the KSPD with those of the BSID-II in a large sample of Japanese infants. The KSPD and BSID-II were administered to 861 7-month-old infants and to 894 18-months old infants. All of the correlation coefficients between the two scales were significant except those between the Language-Social area of the KSPD and Language, Social facets of the BSID-II at 7 months of age. The findings of this study revealed that the results of the KSPD and BSID-II were comparable. There are many reports using the KSPD in Japan, and the findings based of the KSPD and BSID-II may be shared.
\end{abstract}

Key Words: KSPD, BSID-II, developmental assessment tool, psychometrics

\section{Introduction}

Assessing early stages of development is of increased concern worldwide because of the expansion of programs that serve young children with special needs (Bradley-Johnson, 2001). In Japan, several assessment tools have been developed; however, few of those tools can be used to make global comparisons or evaluations (Matsuzaki, Matsui, Ichida, Nakazawa, Hattori, Yoshikoshi, Miyazaki, Fuji, Hagino, Kagisaki, \& Yagihara, 2010).

Among the various tools for assessing developmental stages of infants, the Bayley Scales of Infant Development second edition (BSID-II) (Bayley, 1993) is widely used outside of Japan and is regarded as one of the standards for developmental assessment

\footnotetext{
* Corresponding Author

Mailing Address: 2-1 Seiryo-machi, Aoba-ku, Sendai 980-8575, Japan

E-mail Address: nozomi@med.tohoku.ac.jp

Received October 19, 2012, Accepted April 20, 2013
}

of infants. The BSID-II has been used in studies of preterm infants (Ariagno, Thoman, Boeddiker, Kugener, Constantinou, Mirmiran, \& Baldwin, 1997), infants with prenatal drug exposure (Mayes, Bornstein, Chawarska, \& Granger, 1995; Schuler, Nair, \& Harrington, 2003), and infants with language delay (Campbell, Siegel, Parr, \& Ramey, 1986; Siegel, Cooper, Fitzhardinge, \& Ash, 1995). This scale has also been shown to be sensitive to patterns of developmental changes that are specific to different risk groups of infants. There are some reports on the use of the BSID-II with Japanese infants (Matsuzaki et al., 2010; Nakajima, Saijo, Kato, Sasaki, Uno, Kanagami, Hirakawa, Hori, Tobiishi, Todaka, Nakamura, Yanagiya, Sengoku, Iida, Sata, \& Kishi, 2006; Oka, Suzuki, Nakai, Hosokawa, \& Satoh, 2005; Tanaka, Hosozawa, Kudo, Yoshikawa, Hisata, Shoji, Shinohara, \& Shimizu, 2012). However, the BSID-II has not been standardized in Japan.

The Kyoto Scale of Psychological Development 2001 (KSPD) (Ikuzawa, Iwachidou, \& Oogami, 2001) is a standardized developmental assessment scale for 
Japanese infants. There are many reports on using the KSPD in Japan, but there have been few reports using the English version of KSPD (Kinjo, Ohga, Ochiai, Honjo, Tanaka, Takahata, Ihara, \& Hara, 2011; Kono, Mishina, Sato, Watanabe, \& Honma, 2008; Koyama, Osada, Tsujii, \& Kurita, 2009; Tamaru, Kikuchi, Takagi, Wakamatsu, Ono, Horikoshi, Kihara, \& Nakamura, 2011).

The purpose of this study was to compare the indices of the KSPD with those of the BSID-II. We hypothesized that both scales would yield similar results in spite of their differences in test structures and procedures and, if so, findings based on the two representative scales may be shared. Previously, preliminary data with a small sample was reported on the association between the KSPD and BSID-II for 7-month-old infants (Oka et al., 2005). High correlations were found among the aspects of cognitive and motor functions, whereas low correlations were found among the aspects of language and social functions. In this study, we aimed to readdress the comparison of the indices of the KSPD with those of the BSID-II using a large sample of Japanese infants aged 7 and 18 months.

\section{Methods}

\section{Participants}

This study was conducted as a part of the birth cohort study of the Tohoku Study of Child Development (TSCD) (Nakai, Suzuki, Oka, Murata, Sakamoto, Okamura, Hosokawa, Sakai, Nakamura, Saito, Kurokawa, Kameo, \& Satoh, 2004). From 2001 to 2006, 1,348 healthy pregnant women were registered with their informed consent at obstetrical wards of various hospitals in Tohoku, Japan. All infants born at term (36 to 42 weeks of gestation) without congenital anomalies or diseases were included. Pregnancy and delivery had to be completed without overt signs of serious illness or complications. The Medical Ethics Committee of the Tohoku University Graduate School of Medicine approved the study protocol.

The KSPD and BSID-II were administered to infants of 7 and 18 months old. Of all participants, 1,008 7-month old infants and 1,065 18-month old infants were administered the KSPD and BSID-II, and complete data were available for 861 (457 boys and 404 girls) and 894 (460 boys and 434 girls), respectively.

\section{Instruments}

The KSPD is standardized from neonates to adults. This scale consists of 328 items covering the Cognitive-Adaptive area (C-A), Language-Social area (L$\mathrm{S}$ ), and Postural-Motor area (P-M). From each of the three areas, a sum score is converted to a Developmental Quotient (DQ). Dividing the developmental age by the chronological age and then multiplying the quotient by 100 calculates the Developmental Quotient (DQ). The C-A area assesses non-verbal reasoning and visuospatial perceptions. The L-S area assesses interpersonal relationships, socialization and verbal abilities. The P-M area assesses fine motor functions. For the reliability of administration, the testers were trained and certified by the KSPD center of the Kyoto International Social Welfare Exchange Center, Kyoto, Japan.

The BSID-II has been standardized for subjects ranging from 1 to 42 months of age (Bayley, 1993). This test is categorized into two main domains, the Mental Scale and the Psychomotor Scale. The Mental Scale includes 178 items of increasing difficulty that measure performance in sensory perception, memory, habituation, reasoning, early number concepts, generalization, classification, vocalization, language, and social skills. The Motor Scales includes 111 items that assess the degree of control of the body, coordination of the large muscles, finer manipulatory skills of the hands and fingers, dynamic movement, dynamic praxis, postural imitation, and stereognosis. All items are arranged in sequence of increasing difficulty, representing the maturation of abilities in mental and motor development. The resulting raw scores for each scale are converted into a Mental Developmental Index (MDI) and Psychomotor Developmental Index (PDI), based on their age-appropriate norms. In addition to these overall scores, four subscales (facets) were developed, by having experts designate each item from the MDI and PDI of the following constructs: Cognitive, Language, Social, and Motor facets. The scores of these facets are not standardized and are calculated by adding the number of items scored 'credit.' The same items are often placed in more than one facet. Although the facet scores can be helpful in describing an infant's performance in specific areas, they must be used with caution because the items are not equally represented within facets across the ages. The Cognitive and Motor facets contain many items. In contrast, the Social and Lan- 
guage facets contain few items. Since there is no standardized Japanese version of the BSID-II, a Japanese version was prepared for this study, and its reliability was examined as follows. First, a pediatrician translated the original assessment manual and items into Japanese. The pediatrician had stayed at the University of Rochester School of Medicine (URSM) for several months and performed the BSID-II under the instructions of Professor Philip W. Davidson based on the gold standard of the URSM (Davidson, Myers, Cox, Shamlaye, Choisy, Sloane-Reeves, Cernichiari, Marsh, Berlin, \& Tanner, 1995). Second, we invited a tester from the URSM to Japan to evaluate our testers. Third, we took videos of all the participants when they were assessed. From all participants, 32 videos were selected by random sampling and the tester from the URSM was asked to rescore them for separate evaluation. The results were used to calculate the kappa value for reliability between our scoring and the scoring of the URSM tester.

\section{Procedure}

Perinatal and demographic data including the delivery condition and infant characteristics were obtained from interviews and a questionnaire 4 days after delivery. Evaluations of infant development were performed using the KSPD and the BSID-II when infants were 7 and 18 months old. The participants were invited to the research center at Tohoku University. The examination was performed in a quiet, private room in the presence of the parents. These two assessment scales were administered in randomized order. It took 30-40 min to complete both the KSPD and BSID-II. There are eight common items for 7-month-olds and seven for 18-month-olds between KSPD and BSID-II; for example 'pulls to sitting position,' and 'sits alone steadily' were administered once and scored for each.

\section{Statistical Analysis}

In comparison of the areas of the KSPD with the domains of the BSID-II, the C-A and L-S areas of the KSPD were considered to correspond to the MDI of the BSID-II, and the P-M area of the KSPD was considered to correspond to the PDI of the BSID-II (Oka et al., 2005). Using the facets of the BSID-II, the C-A area of the KSPD corresponded to the Cognitive facet of the BSID-II, the L-S area to the Language and Social facets of the BSID-II, and the P-M area to the Motor facet of the BSID-II.

Pearson's product-moment correlation coefficients $(r)$ were calculated to determine the associations between the scores of the KSPD and BSID-II. Partial correlations $(p r)$ were used to assess these relationships after adjustment for infant gender, testers and age at examination (months). All statistical analyses were performed using SPSS version 17.0 for Windows (SPSS Japan, Tokyo) and statistical significance was set at $p<.05$.

\section{Results}

Table 1 shows the maternal and infant characteristics. The mean age at the 7-month examination was

Table 1 Characteristics of Participants

\begin{tabular}{|c|c|c|c|c|c|c|c|c|}
\hline & \multicolumn{4}{|c|}{7 months old $(n=861)$} & \multicolumn{4}{|c|}{18 months old $(n=894)$} \\
\hline & Mean & $S D$ & Range & $\%$ & Mean & $S D$ & Range & $\%$ \\
\hline \multicolumn{9}{|l|}{ Infant characteristics } \\
\hline Gender (\% girls) & & & & 46.9 & & & & 48.6 \\
\hline Age at examination (month) & 7.0 & 0.2 & $6-9$ & & 18.1 & 0.3 & $17-21$ & \\
\hline Gestational age (week) & 39.6 & 1.2 & $35.6-42.4$ & & 39.6 & 1.2 & $36.0-42.0$ & \\
\hline Birth height $(\mathrm{cm})$ & 49.0 & 1.9 & $31.5-55.0$ & & 48.9 & 1.9 & $31.5-55.0$ & \\
\hline Birth weight (g) & 3114 & 365.0 & $2182-4322$ & & 3105 & 358.6 & $2182-4322$ & \\
\hline Birth order (\% first-born child) & & & & 45.3 & & & & 45.3 \\
\hline \multicolumn{9}{|l|}{ Maternal characteristics } \\
\hline Age at delivery (years) & 30.3 & 4.6 & $16-41$ & & 30.4 & 4.7 & $16-42$ & \\
\hline Education level (\% over 12 years) & & & & 54.6 & & & & 55.7 \\
\hline Delivery type (\% non-spontaneous) & & & & 27.4 & & & & 28.5 \\
\hline
\end{tabular}


7.0 (range, 6-9) months, and that at 18 months was 18.1 (range, 17-21). Table 2 shows the scores of the KSPD and BSID-II. From the score of the KSPD, infants were divided into the normal range, borderline range, and clinical range. Of the 861 infants at 7 months, 852 (99.0\%) were within the normal range, 9 (1.0\%) were within borderline range, and none were in the clinical range. Of the 894 infants at 18 months, $804(89.9 \%)$ were within the normal range, 90 (10.1\%) were within the borderline range, and none were in the clinical range.

\section{BSID-II Interrater Agreement With Gold Standard}

The calculated kappa value for agreement of the BSID-II scores evaluated by local testers and scores evaluated by the tester of the URSM was 0.71 .

\section{Correlation of KSPD Scores With MDI and PDI}

Table 3 shows the results of Pearson's productmoment correlation coefficients and partial corre-

Table 2 Scores on the KSPD and BSID-II

\begin{tabular}{|c|c|c|c|c|c|c|c|c|}
\hline & \multicolumn{4}{|c|}{7 months old $(n=861)$} & \multicolumn{4}{|c|}{18 months old $(n=894)$} \\
\hline & Mean & $S D$ & Min & Max & Mean & $S D$ & Min & $\operatorname{Max}$ \\
\hline \multicolumn{9}{|l|}{ KSPD } \\
\hline $\mathrm{C}-\mathrm{A}$ & 106.3 & 7.0 & 80 & 127 & 97.2 & 9.6 & 72 & 131 \\
\hline $\mathrm{L}-\mathrm{S}$ & 90.6 & 13.4 & 62 & 130 & 97.1 & 11.1 & 59 & 128 \\
\hline P-M & 98.5 & 12.2 & 65 & 139 & 87.2 & 12.8 & 60 & 136 \\
\hline $\mathrm{DQ}$ & 102.7 & 6.7 & 78 & 127 & 95.4 & 8.3 & 73 & 123 \\
\hline \multicolumn{9}{|l|}{ BSID-II } \\
\hline MDI & 93.7 & 5.1 & 73 & 114 & 88.7 & 10.1 & 49 & 117 \\
\hline PDI & 84.1 & 10.7 & 60 & 123 & 85.2 & 10.0 & 49 & 129 \\
\hline \multicolumn{9}{|l|}{ BSID-II facets } \\
\hline Cognitive & 53.0 & 2.2 & 44 & 60 & 83.9 & 3.8 & 57 & 96 \\
\hline Language & 7.3 & 0.9 & 6 & 10 & 20.9 & 3.3 & 12 & 33 \\
\hline Social & 14.9 & 0.3 & 13 & 15 & 16.0 & 0.1 & 15 & 16 \\
\hline Motor & 58.2 & 5.2 & 42 & 79 & 107.1 & 4.1 & 88 & 126 \\
\hline
\end{tabular}

Note. KSPD: Kyoto Scale of Infant Development 2001; C-A, Cognitive-Adaptive; L-S, Language-Social; P-M, Posture-Motor; DQ, Developmental quotient.

BSID-II: Bayley Scales of Infant Development $2^{\text {nd }}$ edition; MDI, Mental Developmental Index; PDI, Psychomotor Developmental Index.

Table 3 Correlation Coefficients Between the KSPD and BSID-II

\begin{tabular}{|c|c|c|c|c|c|c|c|c|}
\hline & \multicolumn{4}{|c|}{ Pearson's correlation coefficient } & \multicolumn{4}{|c|}{ Partial correlation coefficient } \\
\hline & C-A & L-S & $\mathrm{P}-\mathrm{M}$ & DQ & $\mathrm{C}-\mathrm{A}$ & L-S & $\mathrm{P}-\mathrm{M}$ & DQ \\
\hline \multicolumn{9}{|c|}{7 months old } \\
\hline MDI & 0.62 & 0.36 & 0.33 & 0.63 & 0.65 & 0.27 & 0.33 & 0.64 \\
\hline PDI & 0.40 & 0.27 & 0.80 & 0.69 & 0.40 & 0.27 & 0.80 & 0.69 \\
\hline \multicolumn{9}{|c|}{18 months old } \\
\hline MDI & 0.61 & 0.54 & 0.25 & 0.69 & 0.60 & 0.53 & 0.23 & 0.68 \\
\hline PDI & 0.27 & 0.16 & 0.62 & 0.44 & 0.24 & 0.14 & 0.61 & 0.41 \\
\hline
\end{tabular}

Note. Control variables: child gender, age at examination, tester. $p<.01$ for all correlations greater than $r=.11$.

KSPD: Kyoto Scale of Infant Development 2001; C-A, Cognitive-Adaptive; L-S, Language-Social; P-M, Posture-Motor; DQ, Developmental quotient.

BSID-II: Bayley Scales of Infant Development $2^{\text {nd }}$ edition; MDI, Mental Developmental Index; PDI, Psychomotor Developmental Index. 
lation coefficients among KSPD scores (C-A, L-S, $\mathrm{P}-\mathrm{M}$ areas, and DQ) and BSID-II scores (MDI and PDI) for 7- and 18-month-old infants. The C-A area was significantly correlated with the MDI $(r=.62$ at 7 months, and $r=.61$ at 18 months). The L-S area showed a significant correlation with the MDI $(r=.36$ at 7 months, and $r=.54$ at 18 months). The P-M area showed a significant correlation with the PDI ( $r=.80$ at 7 months and $r=.62$ at 18 months). At both ages, the C-A score and the L-S score were significantly correlated with the MDI, whereas the P-M score was significantly correlated with the PDI. After adjustment for confounding factors, similar correlations were found.

\section{Correlations of KSPD Scores With BSID-II Facet Scores}

Table 4 shows the Pearson's product-moment correlation coefficients and the partial correlation coefficients among KSPD scores (C-A, L-S and P-M areas) and BSID-II facet scores (Cognitive, Language, Social and Motor) at 7 and 18 months. The C-A was significantly correlated with the Cognitive facet $(r=.59$ at 7 months and $r=.65$ at 18 months). The L-S area showed a significant correlation with the Language facet ( $r=.39$ at 7 months and $r=.60$ at 18 months). There was no significant relationship between L-S and the Social facet $(r=.12$ at 7 months and $r=.05$ at 18 months). The P-M area showed a significant cor- relation with the Motor facet ( $r=.66$ at 7 months and $r=.54$ at 18 months). Similar results were obtained for the partial correlation coefficients.

\section{Discussion}

The present study was conducted to compare the indices of the KSPD with those of the BSID-II in a large sample of Japanese infants. The correlations between the $\mathrm{DQ}$, which is an overall indicator of the KSPD, and the two domains of the BSID-II were significant at both 7 and 18 months of age. The correlations between the C-A area of the KSPD and MDI/ Cognitive facet of the BSID-II, as well as the P-M area of the KSPD and PDI/Motor facet of the BSIDII, were also significant. Although the L-S area of the KSPD and MDI of the BSID-II correlated significantly, the correlation coefficient was not so high for 7-month old infants.

These two developmental assessment scales have several differences. First, the numbers of assessment items are different. The KSPD consists of fewer items than the BSID-II. Second, they use different categories for classification. The KSPD has three areas; C-A, L-S and P-M. An overall indicator, DQ, is also calculated. In contrast, the BSID-II has two domains, the MDI and PDI, but no overall indicator. Third, the methods used for scoring are different. In the KSPD, the first item to be used is decided by the infants'

Table 4 Correlation Coefficients Between the KSPD and BSID-II Facets

\begin{tabular}{|c|c|c|c|c|c|c|c|c|}
\hline & \multicolumn{4}{|c|}{ Pearson's correlation coefficient } & \multicolumn{4}{|c|}{ Partial correlation coefficient } \\
\hline & $\mathrm{C}-\mathrm{A}$ & $\mathrm{L}-\mathrm{S}$ & P-M & $\mathrm{DQ}$ & $\mathrm{C}-\mathrm{A}$ & L-S & P-M & $\mathrm{DQ}$ \\
\hline \multicolumn{9}{|l|}{7 months old } \\
\hline Cognitive & 0.59 & 0.08 & 0.27 & 0.54 & 0.65 & 0.16 & 0.28 & 0.59 \\
\hline Language & 0.18 & 0.39 & 0.24 & 0.30 & 0.20 & 0.30 & 0.24 & 0.31 \\
\hline Social & 0.10 & 0.12 & 0.00 & 0.10 & 0.09 & 0.07 & -0.01 & 0.08 \\
\hline Motor & 0.48 & 0.20 & 0.66 & 0.65 & 0.55 & 0.22 & 0.72 & 0.74 \\
\hline \multicolumn{9}{|c|}{18 months old } \\
\hline Cognitive & 0.65 & 0.38 & 0.25 & 0.69 & 0.66 & 0.38 & 0.23 & 0.69 \\
\hline Language & 0.37 & 0.60 & 0.17 & 0.49 & 0.36 & 0.60 & 0.16 & 0.48 \\
\hline Social & 0.11 & 0.05 & 0.05 & 0.11 & 0.11 & 0.05 & 0.05 & 0.11 \\
\hline Motor & 0.53 & 0.24 & 0.54 & 0.65 & 0.53 & 0.23 & 0.54 & 0.64 \\
\hline
\end{tabular}

Note. Control variables: child gender, age at examination, tester. $p<.01$ for all correlations greater than $r=.11$.

KSPD: Kyoto Scale of Infant Development 2001; C-A, Cognitive-Adaptive; L-S, Language-Social; P-M, Posture-Motor; DQ, Developmental quotient.

BSID-II: Bayley Scales of Infant Development $2^{\text {nd }}$ edition. 
chronological age. If infants pass the item, they advance to the item corresponding to the next chronological age, but if they cannot perform the item, they stop there. Then the number of items passed is counted and the score is calculated. On the other hand, the BSID-II items follow basal/ceiling rules that have starting and end points that are both based on age and performance. When the basal criterion is not met within the first item set administered, the examiner must go back to the previous item set to establish the basal. Care must be taken in determining the basal and ceiling when more than one item set has to be administered. The basal is established within the lowest item set administered and the ceiling within the highest item set administered. Nevertheless, despite these differences, the results of the KSPD and BSIDII were found to have high correlations among the aspects of cognitive and motor functions. One possible reason for this is that the two developmental assessment tools have the same origin; the BSID-II consists of many items incorporated from Gesell's developmental diagnosis and also from other works such as Kuhlman's Handbook of Mental Tests. Whereas, the KSPD is based on Buhler's test and the Binet test but also on Gesell's developmental diagnosis. Indeed there are eight common items for 7-month-olds and seven for 18-month-olds. Taken together, these areas of the KSPD and BSID-II at 7 and 18 months are considered comparable.

Using the BSID-II facet scores, similar results were found; the correlation coefficient between the L-S area of the KSPD and Language facet of the BSID-II was not high at 7 months. One possible explanation is that the L-S area of the KSPD and Language facet of the BSID-II have very few items (two and five, respectively). On the other hand, the correlation coefficient was increased at 18 months. In the BSID-II, the percentage of language items increases at 18 months (18 items) compared to 7 months (two items). Language is a higher-order cognitive process that plays a central role in the infant's cognitive development and 18-month-old infants have more communication skills than 7-month-olds, so the correlation coefficients increased. Additionally, the correlation coefficient for the L-S area of the KSPD and Social facet of the BSID-II was low. This was probably because both the L-S area of the KSPD (two items for 7 months) and Social facet of the BSID-II (one item for 7 months) consist of very few items. Because there was no item for the Social facet at 18 months, the coefficient of correlation was low. Matsuzaki et al. (2010) compared the BSID-II and the Tsumori test for 12-month-old infants, and they also found a low correlation between cognitive ability and the Language and Social facet of the BSID-II. Thus, the weak correlation coefficients might be related to the number of items of assessment, suggesting that these aspects of the indices are not comparable. Similarly, though the L-S area of the KSPD and MDI of the BSID-II correlated significantly, the correlation coefficient was not so high for 7-month-old infants, whereas that for 18-month-olds was higher. The items constructing the MDI of the BSID-II increases from 19 items for 7 -month-olds to 30 items for 18-month-olds. The value of the correlation coefficient became larger because the number of items increased.

The correlation coefficients of the PDI of the BSIDII and P-M area of the KSPD, and the Motor facet of the BSID-II and the P-M area of the KSPD were lower at 18 months than at 7 months. The correlation $(r=.80)$ of the PDI and P-M at 7 months was high, but decreased to 0.62 at 18 months. One of the possible reasons for this is that the PDI of the BSID-II is a composite of gross motor and fine motor skills. On the other hand, the P-M area of the KSPD consists only of items related to fine motor skills, and there is only one item at 18 months for the P-M area. Thus, correlation was lower at 18 months than at 7 months.

In this study, for reasons of comparability, raw scores of the BSID-II were transformed to yield an average of 100 and a standard deviation of 15 of the US norm. Similar results were obtained using raw scores (data not shown). The mean scores of the BSID-II were lower than 100. The participants of the present study were in the general range because the distributions of the KSPD scores showed normal ranges (Table 2). Since there is no Japanese version of the BSID-II, the MDI and the PDI were calculated using the US norm. Previous studies in Japan showed low mean scores of the MDI and PDI compared with the US norm (Matsuzaki et al., 2010; Nakajima et al., 2006; Tanaka et al., 2012). Similar tendencies have been reported from other counties, including Taiwan (Wu, Tsou, Hsu, Fang, Yao, \& Jeng, 2008), and England (Francis-Williams \& Yule, 1967). The low scores of the BSID-II in the present study might be related to the use of the US norm.

One of the limitations of this study was that the 
two methods were administered only to infants aged 7 and 18 months. Since the BSID-II is standardized for infants between 1 and 42 months old, it is necessary to examine infants at other ages. In Japan, infants receive health checkups at around 6 and 18 months of age. For that reason, we administered the examination at 7 and 18 months. Our results are informative for consideration and comparison with those from other countries. The second limitation is that the BSID-II has been updated to the third edition (BSID-III) (Bayley, 2006). This revision includes separation of the MDI into a distinct cognitive scale, language composite scale with receptive and expressive language subscales, and the PDI into a motor composite scale with fine- and gross-motor subscales. As a result of these changes of the BSID-III, the test structures appear to be more similar to those of the KSPD, which might raise the comparability between the two tests. However, further studies are needed to address the correlation between the two.

In summary, the present study revealed correlations between the KSPD and BSID in 7- and 18-month-old infants. The findings suggest that the $\mathrm{C}-\mathrm{A}$ area of the KSPD and the MDI of the BSID-II, as well as the P-M area of the KSPD and the PDI of the BSID-II, are comparable. There are many reports using the KSPD in Japan these findings based on the KSPD may be compared with those using the BSIDII.

\section{Acknowledgments}

We thank all the families for their participation in the cohort study. We would like to acknowledge all the staff members of Environmental Health Sciences, Tohoku University Graduate School of Medicine, for their help with the organization of data collection for the cohort study.

\section{References}

Ariagno, R. L., Thoman, E. B., Boeddiker, M. A., Kugener, B., Constantinou, J. C., Mirmiran, M., \& Baldwin, R. B. (1997) Developmental care does not alter sleep and development of premature infants. Pediatrics, 100, E9.

Bayley, N. (1993) Bayley Scales of Infant Development second edition. The Psychologial Corporation, San Antonio, Texas.

Bayley, N. (2006) Bayley scales of Infant and Toddler Development (3rd edition). Pearson Assessment Test publishers, San Anto- nio, Texas.

Bradley-Johnson, S. (2001) Cognitive assessment for the youngest children: A critical review of tests. Journal of Psychoeducational Assessment, 19, 19-44.

Campbell, S. K., Siegel, E., Parr, C. A., \& Ramey, C. T. (1986) Evidence for the need to reform the Bayley Scales of Infant Development based on the performance of a population-based sample of 12-month-old infants. Topics in Early Childhood Special Education, 6, 83-96.

Davidson, P. W., Myers, G. J., Cox, C., Shamlaye, C., Choisy, O., Sloane-Reeves, J., Cernichiari, E., Marsh, D. O., Berlin, M., Tanner, M., et al. (1995) Neurodevelopmental test selection, administration, and performance in the main Seychelles child development study. Neurotoxicology, 16, 665-76.

Francis-Williams, J. \& Yule, W. (1967) the bayley infant scales of mental and motor development. An exploratory study with an English sample. Developmental Medicine and Child Neurology, 9, 391-401.

Ikuzawa, M., Iwachidou, S., \& Oogami, R. (2001) The guide of Kyoto Scale of psychological development. In M. Ikuzawa, Y. Matsushita \& A. Nagase (Eds.), Kyoto Kokusai Shakaifukushi Center, Kyoto.

Kinjo, T., Ohga, S., Ochiai, M., Honjo, S., Tanaka, T., Takahata, Y., Ihara, K., \& Hara, T. (2011) Serum chemokine levels and developmental outcome in preterm infants. Early Human Development, 87, 439-443.

Kono, Y., Mishina, J., Sato, N., Watanabe, T., \& Honma, Y. (2008) Developmental characteristics of very low-birth weight infants at 18 months' corrected age according to birth weight. Pediatrics International, 50, 23-28.

Koyama, T., Osada, H., Tsujii, H., \& Kurita, H. (2009) Utility of the Kyoto scale of psychological development in cognitive assessment of children with pervasive developmental disorders. Psychiatry and Clinical Neurosciences, 63, 241-243.

Matsuzaki, T., Matsui, M., Ichida, F., Nakazawa, J., Hattori, A., Yoshikosi, K., Miyazaki, M., Fujii, M., Hagino, I., Kagisaki, K., \& Yagihara, T. (2010) Neurodevelopment in 1-year-old Japanese infants after congenital heart surgery. Pediatrics International, 52, 420-427.

Mayes, L. C., Bornstein, M. H., Chawarska, K., \& Granger, R. H. (1995) Information processing and developmental assessments in 3-month-old infants exposed prenatally to cocaine, Pediatrics, 95, 539-45.

Nakai, K., Suzuki, K., Oka, T., Murata, K., Sakamoto, M., Okamura, K., Hosokawa, T., Sakai, T., Nakamura, T., Saito, Y., Kurokawa, N., Kameo, S., \& Satoh, H. (2004) The Tohoku study of child development: A cohort study of effects of perinatal exposures to methylmercury and environmentally persistent organic pollutants on neurobehavioral development in 
Japanese children. The Tohoku Journal of Experimental Medicine, 202, 227-237.

Nakajima, S., Saijo, Y., Kato, S., Sasaki, S., Uno, A., Kanagami, N., Hirakawa, H., Hori, T., Tobiishi, K., Todaka, T., Nakamura, Y., Yanagiya, S., Sengoku, Y., Iida, T., Sata, F., \& Kishi, R. (2006) Effects of prenatal exposure to polychlorinated biphenyls and dioxins on mental and motor development in Japanese children at 6 months of age. Environmental Health Perspectives, 114, 773-778.

Oka, T., Suzuki, K., Nakai, K., Hosokawa, T., \& Satoh, H. (2005) A trial to apply Bayley scales of infant development second edition to Japanese children. Igaku-no-Ayumi, 212, 259-263. (in Japanese)

Schuler, M. E., Nair, P., \& Harrington, D. (2003) Developmental outcome of drug-exposed children through 30 months: A comparison of Bayley and Bayley-II. Psychological Assessment, $15,435-438$.

Siegel, L. S., Cooper, D. C., Fitzhardinge, P. M., \& Ash, A. (1995) The use of the mental development index of the Bayley scale to diagnose language delay in 2-year-old high-risk infants. Infant Behavior and Development, 18, 483-486.

Tamaru, S., Kikuchi, A., Takagi, K., Wakamatsu, M., Ono, K., Horikoshi, T., Kihara, H., \& Nakamura, T. (2011) Neurodevelopmental outcomes of very low birth weight and extremely low birth weight infants at 18 months of corrected age associated with prenatal risk factors. Early Human Development, 87, $55-59$.

Tanaka, K., Hosozawa, M., Kudo, N., Yoshikawa, N., Hisata, K., Shoji, H., Shinohara, K., \& Shimizu, T. (2013) The pilot study: Sphingomyelin-fortified milk has a positive association with the neurobehavioural development of very low birth weight infants during infancy, randomized control trial. Brain \& Development, 35, 45-52.

Wu, Y. T., Tsou, K. I., Hsu, C. H., Fang, L. J., Yao, G., \& Jeng, S. F. (2008) Brief report: Taiwanese infants' mental and motor development-6-24 months. Journal of Pediatric Psychology, 33, 102-108. 